This item was submitted to Loughborough's Research Repository by the author.

Items in Figshare are protected by copyright, with all rights reserved, unless otherwise indicated.

\title{
Microstrip patch antennas on substrates with metallic inclusions
}

PLEASE CITE THE PUBLISHED VERSION

http://ieeexplore.ieee.org/

PUBLISHER

Loughborough University (@ IEEE)

VERSION

AM (Accepted Manuscript)

LICENCE

CC BY-NC-ND 4.0

REPOSITORY RECORD

Whittow, W.G., Chinwe C. Njoku, and J.C. Vardaxoglou. 2019. "Microstrip Patch Antennas on Substrates with Metallic Inclusions". figshare. https://hdl.handle.net/2134/11525. 
This item was submitted to Loughborough's Institutional Repository (https://dspace.lboro.ac.uk/) by the author and is made available under the following Creative Commons Licence conditions.

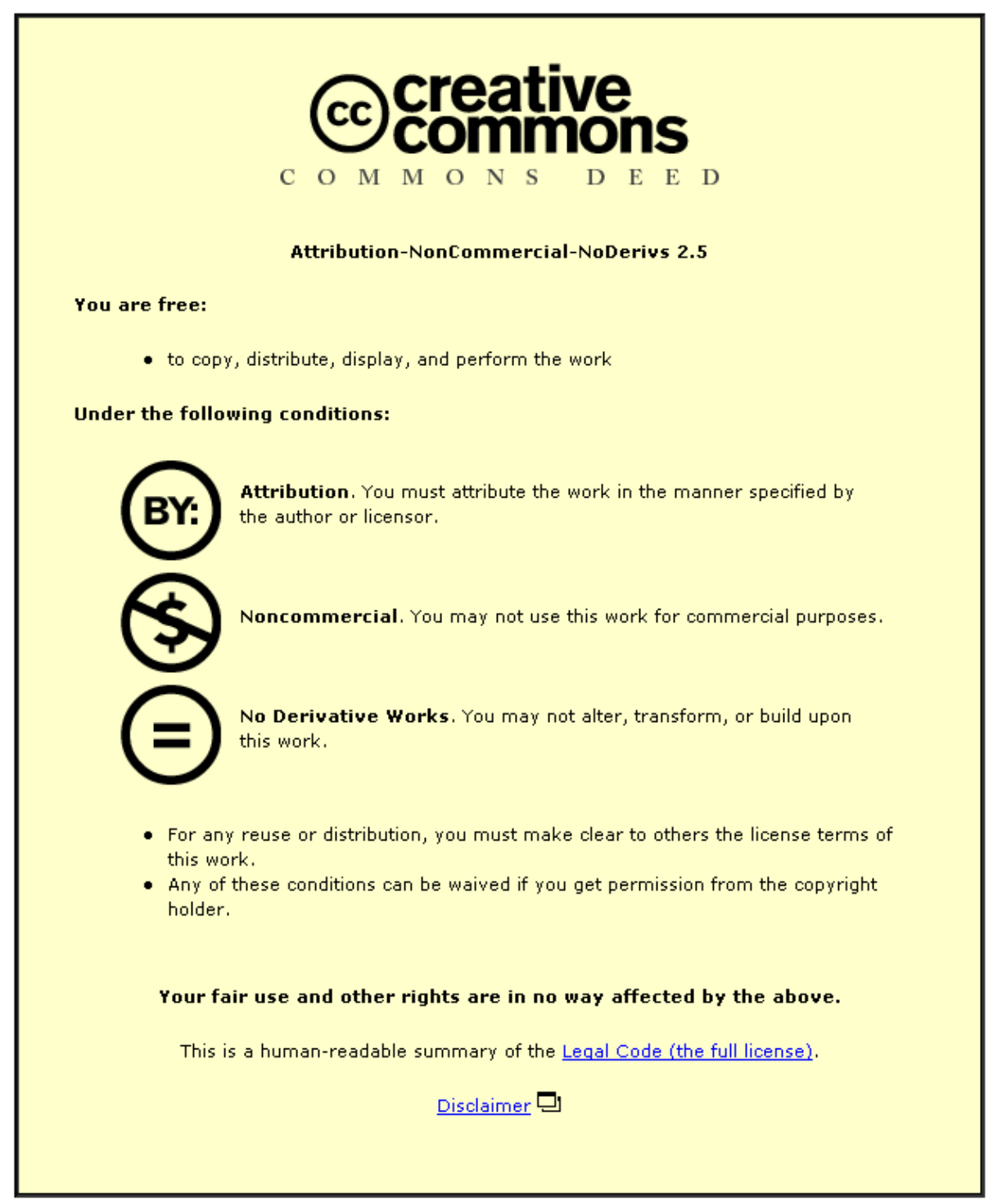

For the full text of this licence, please go to: http://creativecommons.org/licenses/by-nc-nd/2.5/ 


\title{
Microstrip Patch Antennas on Substrates with Metallic Inclusions
}

\author{
C. C. Njoku, W. G. Whittow and J. C. Vardaxoglou \\ School of Electronic, Electrical and Systems Engineering, Loughborough University, Loughborough, LE11 3TU, UK \\ j.c.vardaxoglou@lboro.ac.uk
}

\begin{abstract}
This paper considers the design of microwave patch antennas using bespoke substrates. The permittivity of the substrate is controlled by inserting small scale metallic inclusions into a host medium and can be obtained using an S-parameter inversion algorithm on the results of plane-wave simulations. Electromagnetic simulations have been used to compare the performance of the patch on the heterogeneous substrate to standard homogeneous substrate.
\end{abstract}

Keywords- artificial dielectrics, effective media, heterogeneous mixtures, patch antennas

\section{INTRODUCTION}

Antennas typically consist of metallic and insulating materials. Several papers have investigated artificial dielectric materials [1-8]. Synthetic materials removes the limitation of having to work with existing materials and allows the 'creation' of new materials that can be designed to have the required constitutive parameters, losses and other characteristics pre-determined for the whole antenna system.

In 1946, Lewin analytically calculated the effective permittivity and permeability of a material loaded with small spherical particles [7]. This seminal work forms a basis for a combination of different kinds of structures with bespoke electromagnetic (EM) parameters.

Technology and fabrication processes have made rapid advances in the last 60 years and nanotechnology [9], [10] can offer exciting new opportunities in terms of constructing new materials with amazing properties.

These bespoke materials can be integrated to form complete antennas systems where the metallic and dielectric elements are made in one process, resulting in time and potential cost savings [11], [12], [13].

Patch antennas [14], [15] are currently fabricated using destructive processes where a copper film is printed across the whole surface and then the undesired copper is etched away using environmentally damaging chemicals.

Controlling the permittivity allows an extra degree of freedom for antenna designers. Previous work has shown that higher bandwidths can be achieved by varying the permittivity of the substrate as a function of location [16]. It was hypothesized that further improvements could be obtained by using a smooth transition between local dielectric regions as could be achieved with artificial dielectrics [17-19]. A small size, high efficiency antenna can be achieved by using an as yet unrealizable material with equality of permittivity and permeability [20]. An initial study by the authors gave preliminary insight into the behaviour of patch antennas on synthetic media with dielectric inclusions [11], [21].

Section II calculates the real and imaginary effective permittivity of small metallic inclusions embedded in a host medium using an inversion algorithm process on the scattering parameters from plane waves impinging on the heterogeneous medium of different thicknesses. Once these are known, patch antennas with heterogeneous substrates can then be compared with a homogenous patch. A transmission line analysis is given in Section III while the patch antenna results are analysed in Section IV and conclusions are highlighted in Section V.

\section{Plane Wave Analysis}

It is well known that the electrical properties of a material can be understood by the way it responds to a plane wave (PW) travelling through it. Based on this, the scattering parameters, $S_{11}$ and $S_{21}$, and the thickness of the material, when processed using suitable inversion processes, for example as shown in [22-24], gives its electrical properties such as the relative permittivity, $\varepsilon_{r}$ and permeability, $\mu_{r}$. The main property being examined here is the relative permittivity of the material, in this case, a heterogeneous medium within which are embedded metallic cubes in a rectangular lattice, as shown in Fig. 1. In a previous paper [25], the authors have shown that the use of metallic cubes can increase the relative permittivity of the host material by a factor of over 20 . The values of permittivity and permeability in this paper are relative values. 
Final author version. Paper published at Loughborough Antennas \& Propagation Conference, Loughborough UK, 2012. The paper is also available on IEEE Xplore

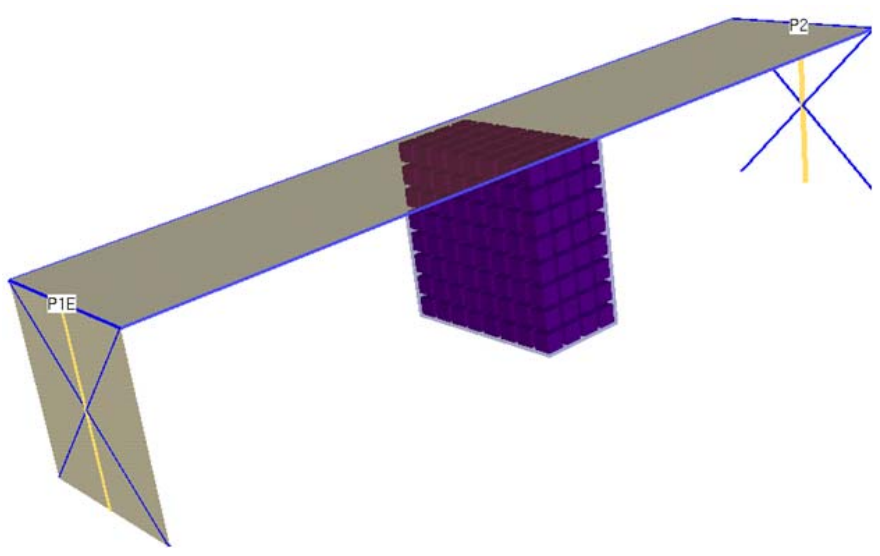

Figure 1. Simulation set-up of a 4-layer structure of metallic cubes in a cubic lattice within a homogenous host

The ports were positioned at each end. Perfect Electric Conductor (PEC) and Perfect Magnetic Conductor (PMC) boundary conditions were used along two axes to replicate a semi-infinite structure, while the structure was finite in the direction of propagation. The $S_{11}$ and $S_{21}$ are passed through the "Inverse Scattering Formalism" after a phase rectification process [23] from which the effective permittivity, $\varepsilon_{\text {eff }}$ and permeability, $\mu_{\text {eff }}$ are extracted. Further details can be found in [11]. One layer of cubes (equal to unit cell size) is $0.5 \mathrm{~mm}$.

Data used: cube size, $a=395 \mu \mathrm{m}$, inclusions' periodicity, $s$ $=500 \mu \mathrm{m}$, host permittivity, $\varepsilon_{1}=2.25$ (Polyethylene), inclusions' permittivity, $\varepsilon_{2}=\left(1.27-\mathrm{i}^{*} 103.53\right) * 10^{6}$ (Copper, obtained from the Drude model [26]), host permeability, $\mu_{1}=$ inclusions'permeability, $\mu_{2}=1$.

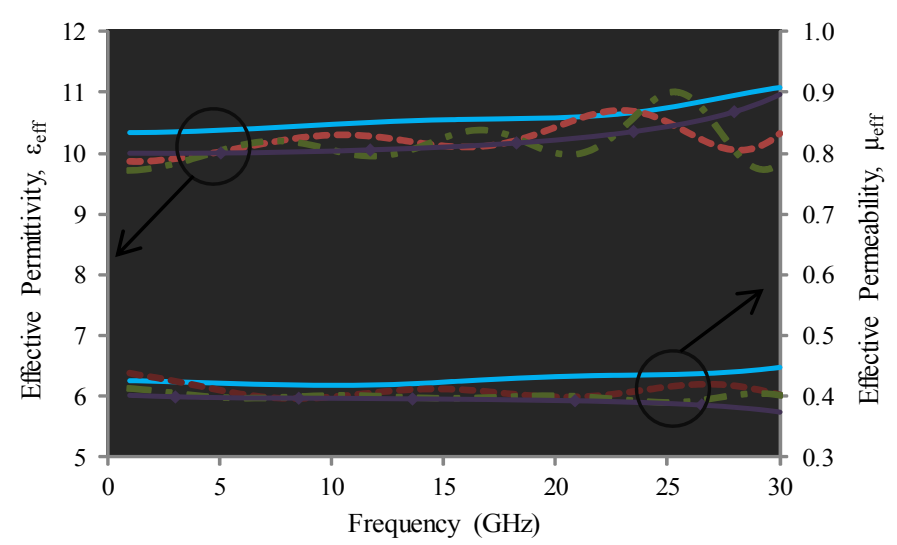

Figure 2. Effective permittivity, $\varepsilon_{e f f}$ and permeability, $\mu_{\text {eff }}$ of a 1-layer (-), 2-layer (----), 3-layer ( $-\cdot-$ ) and 4-layer ( $-\bullet)$ heterogeneous medium of copper cubes in a homogenous dielectric host

For comparison, the $\varepsilon_{\text {eff }}$ from the different number of layers are shown in Fig. 2. As shown the value of the $\varepsilon_{\text {eff }}$ tends to reduce as the number of layers increase. Table I lists these results from the PW simulations and inversion process an average of the $\varepsilon_{\text {eff }}$ over the frequency range. The analytical equations [7] produced an effective permittivity of 8.82 using spheres of the same volume fraction. The difference in $\varepsilon_{e f f}$

may be due to the equations in [7] being based on spherical inclusions and an infinite medium, also seen in [25].

TABLE I. AVERAGE $\varepsilon_{e f f}$ RESULTS FROM FIG. 2

\begin{tabular}{|c|c|c|}
\hline $\begin{array}{c}\text { Number of } \\
\text { Layers }\end{array}$ & $\begin{array}{c}\text { Substrate } \\
\text { thickness, } \mathrm{mm}\end{array}$ & $\begin{array}{c}\varepsilon_{\text {eff }}(\mathrm{PW} \\
\text { simulations })\end{array}$ \\
\hline 1 & 0.5 & 10.58 \\
\hline 2 & 1.0 & 10.24 \\
\hline 3 & 1.5 & 10.17 \\
\hline 4 & 2.0 & 10.21 \\
\hline
\end{tabular}

\section{TRANSMISSION LINE SIMULATIONS}

A transmission line of the same width was placed on the heterogeneous (with equally spaced $395 \mu \mathrm{m}$ metallic cubes) and homogenous equivalent $\left(\varepsilon_{\text {eff }}=10.24\right)$ of $1 \mathrm{~mm}$ thickness; see Fig. 3 (a) and (b). Their resulting impedances were compared as a preliminary study to the analysis of patch antenna structures. Fig. 3(c) shows the plots of the real and imaginary parts of these impedances.

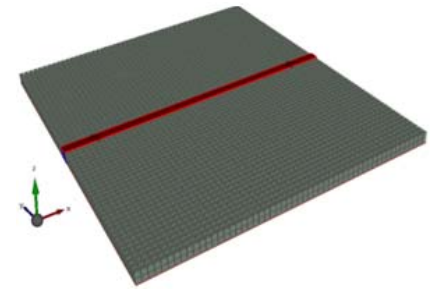

(a)

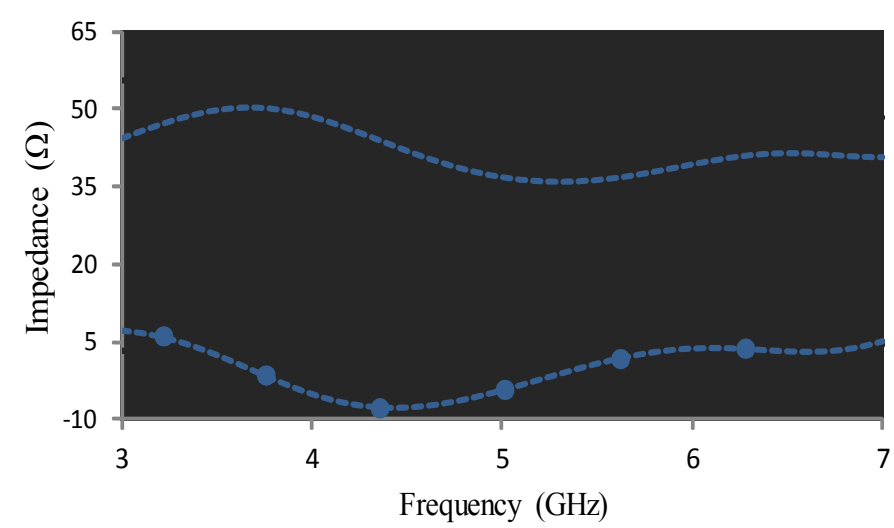

(c)

Figure 3. $870 \mu \mathrm{m}$ wide transmission line on a (a) heterogeneous and (b) homogenous substrate; (c) Real (unmarked) and imaginary (marked) parts of input impedance of a transmission line on a homogenous (-) and heterogeneous (------) substrate

As shown, the impedance and the S-parameters $\left(S_{11}\right.$ and $S_{21}$ ) of the homogenous varies more with frequency than that of the heterogeneous over the frequency range. The impedance of transmission line on the heterogeneous substrate is close to $50 \Omega$ and indicates that the effective permittivity approximates the homogeneous version.

This work was supported by EPSRC under Grant No. EP/I01490X/1 
Final author version. Paper published at Loughborough Antennas \& Propagation Conference, Loughborough UK, 2012. The paper is also available on IEEE Xplore

\section{PATCH ANTENNA SimUlations}

In [11], the authors showed that the performance of a patch antenna on a heterogeneous medium with equally spaced dielectric spheres in a dielectric host agreed well with the patch on a homogenous substrate of the same $\varepsilon_{e f f}$. $\varepsilon_{\text {eff }}$ is obtained by taking the mean over the frequency range of the permittivity from the inversion process. The patch dimensions used in this section are $11.83 \mathrm{~mm}$ by $19.97 \mathrm{~mm}$. The substrate thickness and position of the offset feed were the same for both the homogenous and heterogeneous case. Fig. 4 shows an example of the patch on the homogenous and heterogeneous media.

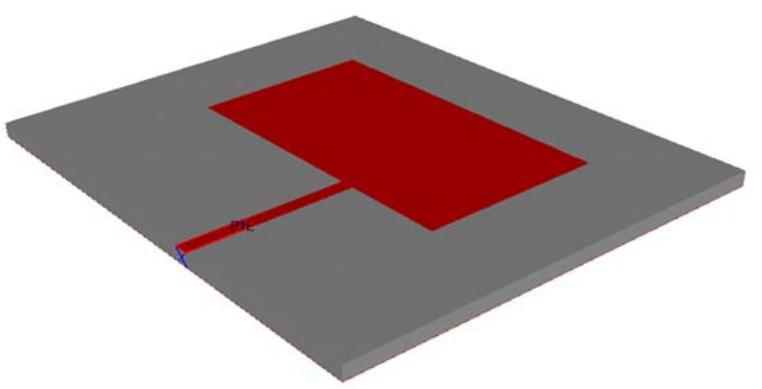

(a)

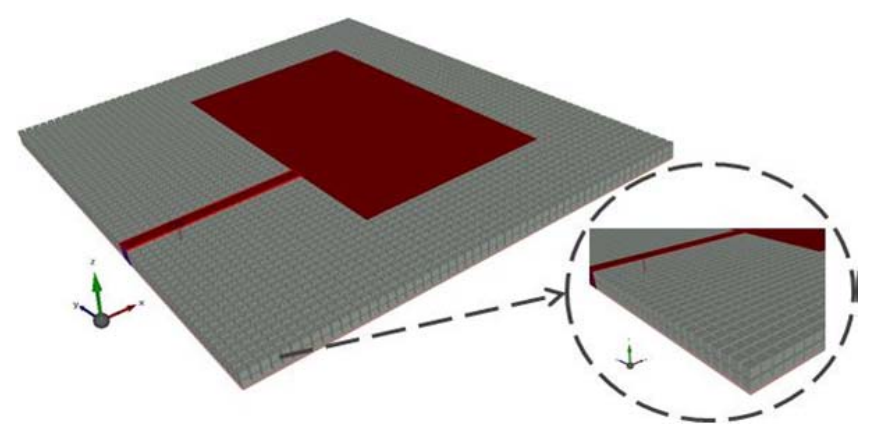

(b)

Figure 4. Offset feed rectangular patch on a (a) homogenous and (b) heterogeneous substrate

Initially, dielectric inclusions were used with their permittivity increased from 10 to 110 with the same patch size, feed position and substrate thickness, $1 \mathrm{~mm}$. The $S_{11}$ results are shown in Fig. 5. As expected, the resonant frequency decreases as the permittivity of the cubic inclusions increases.

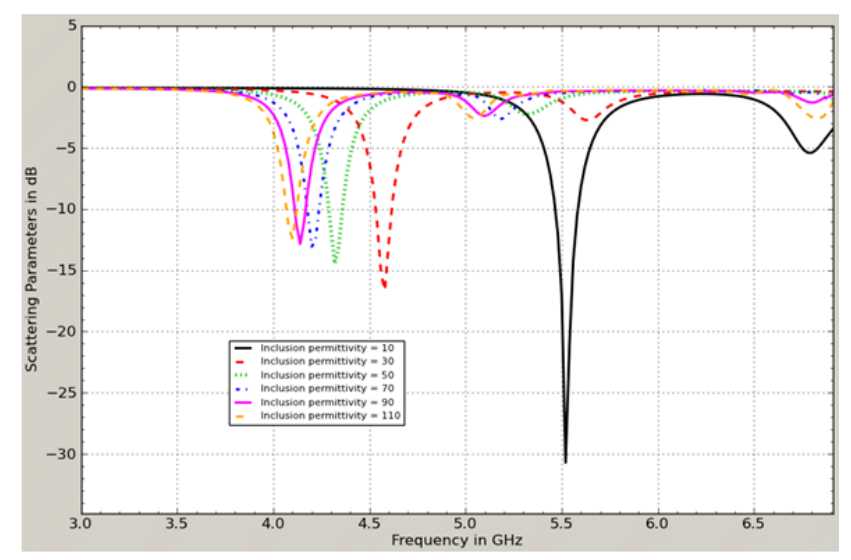

Figure 5. Return loss of patch antenna with dielectric cubes of increasing permittivity in a low dielectric substrate, $\varepsilon_{r}=2.25$.

As the average values of $\varepsilon_{\text {eff }}$ for each number of layers are very similar, the same offset-fed patch size was used. The same data in Section II was used for the substrate with metallic inclusions. The effective $\varepsilon_{r}$ used for the homogenous substrate is as shown in Table I. The $S_{11}$ results of the n-layered media and their homogenous equivalents are shown in Fig. 6.

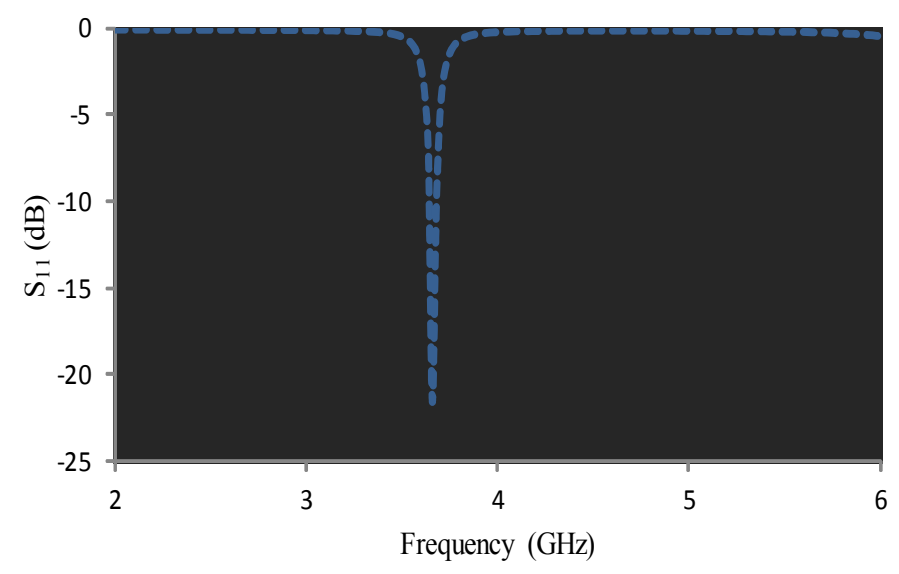

(a)

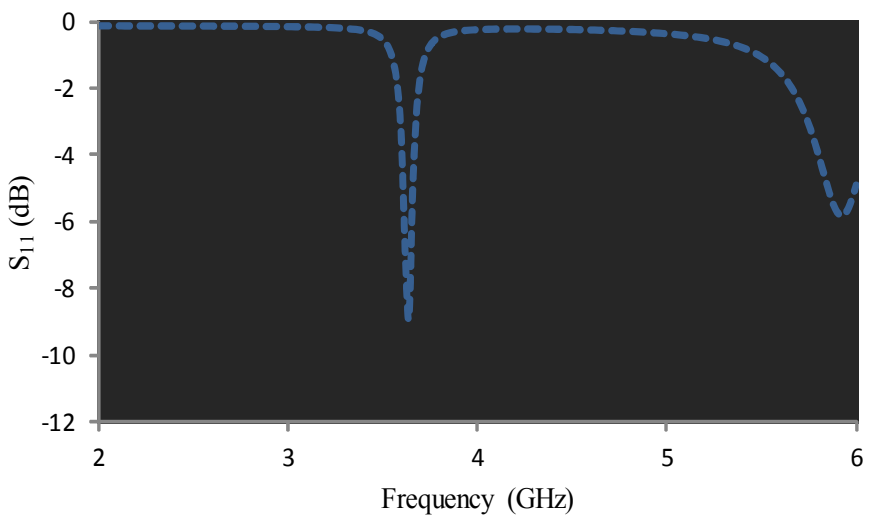

(b) 
Final author version. Paper published at Loughborough Antennas \& Propagation Conference, Loughborough UK, 2012. The paper is also available on IEEE Xplore

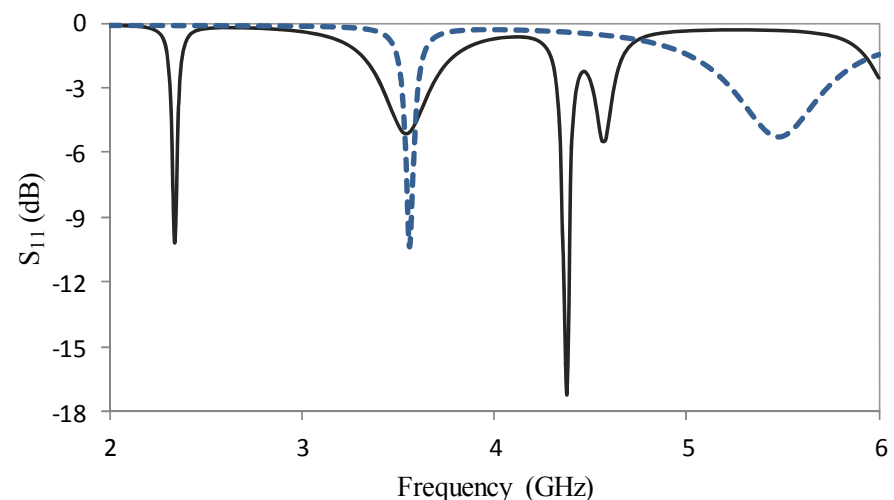

(c)

Figure 6. Return loss $\left(S_{11}\right)$ of a rectangular patch antenna on a homogenous equivalent (-) and heterogeneous (--- --) substrate for (a) $0.5 \mathrm{~mm}$ thick (or 1layer of cubes in the heterogeneous case), (b) $1 \mathrm{~mm}$ thick (2-layered) and (c) $1.5 \mathrm{~mm}$ thick (3-layered)

From Fig. 6, the resonance frequency for both antennas is just less than $4 \mathrm{GHz}$. Further work is needed to fully understand the differences. By using the equations for a designing a patch antenna [15], it can be easily worked out that the permittivity being 'seen' by the patch is around 10 - as predicted by the PW process. The resonant frequencies of the patch antennas on the heterogeneous and the homogenous equivalent substrates are shown in Table II.

TABLE II. FREQUENCY OF PATCH ANTENNAS

\begin{tabular}{|c|c|c|c|}
\hline & \multicolumn{2}{|c|}{ Homogenous } & $\begin{array}{c}\text { Heterogeneous (with } \\
1,2,3 \text { layers of metal } \\
\text { cubes) }\end{array}$ \\
\hline $\begin{array}{c}\text { Substrate } \\
\text { thickness } \\
(\mathrm{mm})\end{array}$ & $\begin{array}{c}\text { Permittivity } \\
\text { used for } \\
\text { homogeneous } \\
\text { substrate }\end{array}$ & $f_{r}(\mathrm{GHz})$ & $f_{r}(\mathrm{GHz})$ \\
\hline 0.5 & 10.58 & 3.94 & 3.66 \\
\hline 1.0 & 10.24 & 3.77 & 3.64 \\
\hline 1.5 & 10.17 & 3.54 & 3.56 \\
\hline
\end{tabular}

\section{CONCLUSIONS}

In this paper, we have investigated the concept of designing artificial dielectrics using composite metallic and non-metallic materials. The volume density of small metallic inclusions can be used to control the effective permittivity of the mixture. This has been demonstrated using plane wave simulations. Simulated transmission line results have also indicated that the effective permittivity of the mixture is as predicted by analytic theory. Furthermore, simulations with patch antenna substrates with inserted dielectric cubes behave as expected. The agreement is less clear when patch antennas are placed above heterogeneous substrates containing metal cubes. The frequency response did not match the equivalent homogeneous case and the radiation efficiency was lower. This is thought to be due to the patch coupling to the metal cubes and the surface waves along the heterogeneous substrate. It is hypothesized that this may be improved by using smaller cubes sizes.

Future work will decrease the size of the cubes towards the nano-scale to increase the isotropy. Fabricating these structures is not straightforward and we are in discussions with colleagues to create suitable samples which will then allow measurements.

\section{REFERENCES}

[1] W. T. Doyle, "The Clausius-Mossotti problem for cubic array of spheres", Journal of Applied Physics, vol. 49, issue 2, pp. 795-797, Feb. 1978

[2] W. T. Doyle, "Optical properties of a suspension of metal spheres", Physics Review B, vol. 39, issue 14, pp. 9852-9858, May 1989.

[3] R. E. Collin, Ch. 12 in Field Theory of Guided Waves, $2^{\text {nd }}$ edition, IEEE Press: NY, 1991

[4] A. Sihvola, Ch. 3 in Electromagnetic mixing formulas and applications, IEE Electromagnetic Waves Series 47, 1999

[5] I. A. Kolmakov, L. Jylha, S. A. Tretyakov, and S. Maslovski, "Lattice of dielectric particles with double negative response", 28th General Assembly of International Union of Radio Science (URSI), New Delhi, India, paper BCD.5 (0109).pdf, 2005

[6] W. G. Whittow, and J. C. Vardaxoglou, "Modifying conventional microwave antenna designs using fine scale structures and nanomaterials," LAPC 2009, pp. 749-752, Dec. 2009

[7] L. Lewin, "The electrical constants of a material loaded with spherical particles", IEE - Part III: Radio and Communication Engineering, vol. 94, issue 27, pp. 65-68, Jan. 1947

[8] L. Rayleigh, "On the influence of obstacles arranged in rectangular order on the properties of a medium," Phil. Mag., ser. 5, vol. 34, pp.481-502, 1892

[9] YouTube Video - "IBM Self Assembly Technology Creates Airgap Microprocessors." (2007, May 3). Available: http://www.youtube.com/watch? $\mathrm{v}=\mathrm{T} 8 \mathrm{p} 8$ zZNJEU

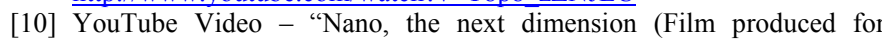
European Commission)." (2008, March 9). Available: http://www.youtube.com/watch?v=eCpkq AeX50

[11] C. C. Njoku, W. G. Whittow, and Y. C. Vardaxoglou, "Simulation methodology for synthesis of antenna substrates with micro-scale inclusions," IEEE Trans. on Antennas and Propagation, vol. 60, issue 5, pp. 2194-2202, May 2012.

[12] F. Juillerat, H. H. Solak, P. Bowen, and H. Hofmann, "Fabrication of large-area ordered arrays of nanoparticles on patterned substrates", Inst. Of Phys. Pub., Nanotech., vol. 16, no. 8, pp.1311-1316, June 2005.

[13] C. Hsu, and Y. Lee, "Fabrication of flexible nano-wired polarizer by contact-transferred and mask embedded lithography and polyurethane acrylate method", Proc. $5^{\text {th }}$ IEEE Int. Conf. NEMS, Xiamen, China, 2010, pp. 893-897.

[14] J.R. James and P. S. Hall, Handbook of Microstrip Antennas, IET, 1989.

[15] R. Garg, Ch. 4 in Microstrip Antenna Design Handbook, Artech House, 2001.

[16] G. Kiziltas, D. Psychoudakis, J. L. Volakis, and N. Kikuchi, “Topology design optimization of dielectric substrates for bandwidth improvement of a patch antenna", IEEE Trans. Antennas Propagat., vol. 51, issue 10, pp. 2732-2743, Oct. 2003

[17] D. Psychoudakis, Y. H. Koh, J. L. Volakis, and J. H. Halloran, "Design method for aperture-coupled microstrip patch antennas on textured dielectric susbtrates," IEEE Trans. on Antennas and Propagation, vol. 52, no. 10 , pp. 2763-2766, 2004.

[18] W. G. Whittow, C. C. Njoku, J. C. Vardaxoglou, and J. Joubert, "Designing multi-band and high bandwidth antennas with heterogeneous substrates," IEEE Tropical Conference on Antennas and Propagation in Wireless Communications (APWC), 2012.

[19] C. C. Njoku, W. G. Whittow and J. C. Vardaxoglou, "Microwave antennas and heterogeneous substrates using nanomaterial fabrication techniques (Invited Paper)," in ," IEEE Tropical Conference on Antennas and Propagation in Wireless Communications (APWC), 2011. 
Final author version. Paper published at Loughborough Antennas \& Propagation Conference, Loughborough UK, 2012. The paper is also available on IEEE Xplore

[20] M. I. Kitra, C. J. Panagamuwa, P. McEvoy, J. C. Vardaxoglou, and J. R. James, "Low SAR ferrite handset antenna design", IEEE Trans. Antennas Propagat., vol. 55, issue 4, pp. 1155-1164, April 2007.

[21] C. C. Njoku, W. G. Whittow and J. C. Vardaxoglou, "Antenna performance on quasi synthetic media," IEEE Intl Workshop Antenna Technology (IWAT), 2012

[22] D. R. Smith, D. C. Vier, Th. Koschny, and C. M. Soukolis, "Electromagnetic Parameter Retrieval from Inhomogeneous Metamaterials", Physical Review E71, 036617, 2005.

[23] N. G. Alexopoulos, C. A. Kyriazidou, and H. F. Contopanagos, "Effective Parameters for Metamorphic Materials and Metamaterials
Through a Resonant Inverse Scattering approach," IEEE Transactions on Microwave Theory and Techniques, vol. 55, no. 2, Feb. 2007.

[24] W. B. Weir, "Automatic Measurement of Complex Dielectric Constant and Permeability at Microwave Frequencies," Proceedings of the IEEE, vol. 62, no. 1, pp. 33-36, Jan. 1974.

[25] C. C. Njoku, W. G. Whittow and J. C. Vardaxoglou, "Effective Permittivity of Heterogeneous Substrates with Cubes in a 3-D Lattice," IEEE AWPL, vol. 10, 2011, pp. 1480-1483.

[26] M. A. Ordal, et al., "Optical properties of the metals $\mathrm{Al}, \mathrm{Co}, \mathrm{Cu}, \mathrm{Au}, \mathrm{Fe}$, $\mathrm{Pb}, \mathrm{Ni}, \mathrm{Pd}, \mathrm{Pt}, \mathrm{Ag}, \mathrm{Ti}$, and $\mathrm{W}$ in the infrared and far infrared", Applied Optics, vol. 22, no. 7, April 1983. 